1 Hacettepe Journal of Mathematics and Statistics

$\bigcap$ Volume 45 (4) (2016), 1135-1142

\title{
The Zagreb coindices of a type of composite graph
}

\author{
Lihui Yang*, Xiaowei $\mathrm{Ai}^{\dagger}$ and Lin Zhang ${ }^{\ddagger}$
}

\begin{abstract}
For a nontrivial graph $G$, its first and second Zagreb coindices are defined as the sum of degree sum of of nonadjacent vertex pairs and the sum of degree product of nonadjacent vertices pairs, respectively. Motivated by the work in [1], we study Zagreb coindices of a new kind of composite graph, namely, double graph. For any given nontrivial graph, explicit formulas are given for the Zagreb coindices of its double graph and $k$-iterated double graph, respectively.
\end{abstract}

Keywords: Degree; Zagreb indices; Zagreb coindices; double graph; $k$-iterated double graph.

2000 AMS Classification: 05C35; 05C12; 05C90.

Received : 30.12.2011 Accepted : 20.01.2013 Doi : 10.15672/HJMS.20164520079

\section{Introduction}

Let $G$ be a simple graph with vertex set $V(G)$ and edge set $E(G)$. For a graph $G$, we let $d_{G}(v)$ be the degree of a vertex $v$ in $G$, i.e., the number of the first neighbors of vertex $v$.

A topological index or graph invariant is a function defined on a (molecular) graph regardless of the labeling of its vertices. Till now, hundreds of different graph invariants have been employed in QSAR/QSPR studies, some of which have been proved to be successful (see [11]). Among those successful invariants, there are two topological indices,

\footnotetext{
${ }^{*}$ College of Mathematics and Computer Science, Hunan City University, Yiyang City, Hunan 413000, China

Email : 1lhhyang@yeah.net

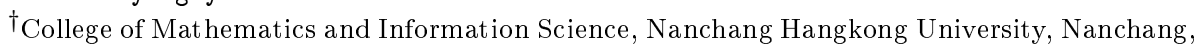
Jiangxi 330063, P.R. China

$\ddagger$ College of Mathematics and Information Science, Nanchang Hangkong University, Nanchang, Jiangxi 330063, P.R. China
} 
relevant to our paper, called the first Zagreb index and the second Zagreb index (see $[3,4,8,10,14])$, defined as

$$
M_{1}(G)=\sum_{u \in V(G)}\left[d_{G}(u)\right]^{2} \text { and } M_{2}(G)=\sum_{u v \in E(G)} d_{G}(u) d_{G}(v)
$$

respectively.

Equivalently, we can rewrite the first Zagreb index as

$$
M_{1}(G)=\sum_{u v \in E(G)}\left[d_{G}(u)+d_{G}(v)\right]
$$

More recently, the authors [2] proposed two new Zagreb-type indices, namely, the first Zagreb coindex and second Zagreb coindex as

$$
\bar{M}_{1}(G)=\sum_{u v \notin E(G)}\left[d_{G}(u)+d_{G}(v)\right] \text { and } \bar{M}_{2}(G)=\sum_{u v \notin E(G)} d_{G}(u) d_{G}(v),
$$

respectively.

It is well-known that one can construct many graphs from simpler graphs via various graph operations. Thus, it is important to understand how certain invariants of such composite graphs are related to the corresponding invariants of the original graphs.

More recently, Ashrafi et al. [1] investigated Zagreb coindices and presented explicit formulas for these new graph invariants under several graph operations, including union, join, Cartesian product, disjunction product, etc. Ashrafi et al. [2] determined the extremal values of Zagreb coindices over some special classes of graphs. Hua and Zhang [5] revealed some relations between Zagreb coindices and some other distance-based topological indices.

The double graph (see [9]) $G^{*}$ of a given graph $G$ is constructed by making two copies of (including the initial edge set of each) and adding edges $u_{1} v_{2}$ and $u_{2} v_{1}$ for every edge $u v$ of $G$. For a nontrivial graph $G$, its $k$-iterated double graph $G^{k *}$, is defined as

$$
G^{1 *}=G^{*} \text { and } G^{k *}=\left(G^{(k-1) *}\right)^{*} \text { for } k \geq 2 .
$$

In particular, it is generally assumed that $G^{0 *}=G$ for the sake of consistence.

For results on double graphs, see $[6,7,12,13]$ and the references cited therein.

Motivated by the work in [1], we study Zagreb coindices of double graph. For any given nontrivial graph, explicit formulas are given for the Zagreb coindices of its double graph and $k$-iterated double graph, respectively.

\section{Main results}

We begin with some notation and terminology used in the proof of our results.
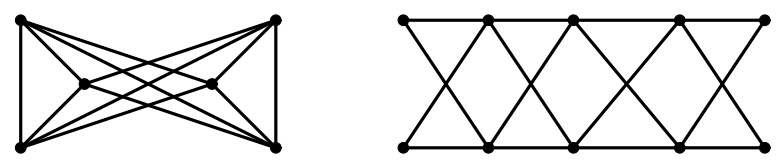

Fig. 1. The double graphs of $C_{3}$ and $P_{5}$.

For each vertex $u$ in a nontrivial graph $G$, we call the corresponding vertices $u_{1}$ and $u_{2}$, in $G^{*}$, the clone vertices of $u$. As examples, we depicted the double graphs $C_{3}^{*}$ (Fig. $1(\mathrm{a}))$ and $P_{5}^{*}$ (Fig. 1(b)) of $C_{3}$ (Fig. 1(a)) and $P_{5}$, respectively. 
For a given vertex $v$ in $G$, if we let $\bar{D}_{G}^{1}(v)=\sum_{u v \notin E(G)}\left[d_{G}(u)+d_{G}(v)\right]$ and $\bar{D}_{G}^{2}(v)=$ $\sum_{u v \notin E(G)} d_{G}(u) d_{G}(v)$, then we can rewrite the expressions of $\bar{M}_{1}(G)$ and $\bar{M}_{2}(G)$ as

$$
\bar{M}_{1}(G)=\frac{1}{2} \sum_{v \in V(G)} \bar{D}_{G}^{1}(v)
$$

and

$$
\bar{M}_{2}(G)=\frac{1}{2} \sum_{v \in V(G)} \bar{D}_{G}^{2}(v),
$$

respectively.

Similarly, if we denote $D_{G}^{2}(v)=\sum_{u v \in E(G)} d_{G}(u) d_{G}(v)$, then the second Zagreb index of $G$ can be rewritten as

$$
M_{2}(G)=\frac{1}{2} \sum_{v \in V(G)} D_{G}^{2}(v) .
$$

In the following, we shall state and prove our main results of this paper.

2.1. Theorem. Let $G$ be a nontrivial graph of order $n$ and size $m$. Then

(i) $\bar{M}_{1}\left(G^{*}\right)=8 \bar{M}_{1}(G)+8 m$;

(ii) $\bar{M}_{2}\left(G^{*}\right)=8 \bar{M}_{2}(G)-8 M_{2}(G)+16 m^{2}$.

Proof. For the sake of convenience, we label all vertices in $G$ as $\left\{v_{1}, \ldots, v_{n}\right\}$. Suppose that $x_{i}$ and $y_{i}$ are the corresponding clone vertices, in $G^{*}$, of $v_{i}$ for each $i=1, \ldots, n$. For any given vertex $v_{i}$ in $G$ and its clone vertices $x_{i}$ and $y_{i}$, there exists $d_{G^{*}}\left(x_{i}\right)=$ $d_{G^{*}}\left(y_{i}\right)=2 d_{G}\left(v_{i}\right)$ by the definition of double graph.

For $v_{i}, v_{j} \in V(G)$, if $v_{i} v_{j} \notin E(G)$, then $x_{i} x_{j} \notin E(G), y_{i} y_{j} \notin E(G), x_{i} y_{j} \notin E(G)$ and $y_{i} x_{j} \notin E(G)$.

So we need only to consider total contribution of the following three types of nonadjacent vertex pairs both to $\bar{M}_{1}\left(G^{*}\right)$ and to $\bar{M}_{2}\left(G^{*}\right)$.

- Type 1: The nonadjacent vertex pairs $\left\{x_{i}, x_{j}\right\}$ and $\left\{y_{i}, y_{j}\right\}$, where $v_{i} v_{j} \notin E(G)$.

- Type 2: The nonadjacent vertex pairs $\left\{x_{i}, y_{i}\right\}$ for each $i=1, \ldots, n$.

- Type 3: The nonadjacent vertex pairs $\left\{x_{i}, y_{j}\right\}$ and $\left\{y_{i}, x_{j}\right\}$, where $v_{i} v_{j} \notin E(G)$.

The total contribution of nonadjacent vertex pairs of type 1 to $\bar{M}_{1}\left(G^{*}\right)$ and $\bar{M}_{2}\left(G^{*}\right)$ are, respectively, given by

$$
\begin{aligned}
\sum_{y_{i} y_{j} \notin E\left(G^{*}\right)}\left[d_{G^{*}}\left(y_{i}\right)+d_{G^{*}}\left(y_{j}\right)\right] & =\sum_{x_{i} x_{j} \notin E\left(G^{*}\right)}\left[d_{G^{*}}\left(x_{i}\right)+d_{G^{*}}\left(x_{j}\right)\right] \\
& =\sum_{v_{i} v_{j} \notin E(G)}\left[2 d_{G}\left(v_{i}\right)+2 d_{G}\left(v_{j}\right)\right] \\
& =2 \sum_{v_{i} v_{j} \notin E(G)}\left[d_{G}\left(v_{i}\right)+d_{G}\left(v_{j}\right)\right] \\
& =2 \bar{M}_{1}(G)
\end{aligned}
$$


and

$$
\begin{aligned}
\sum_{y_{i} y_{j} \notin E\left(G^{*}\right)} d_{G^{*}}\left(y_{i}\right) d_{G^{*}}\left(y_{j}\right) & =\sum_{x_{i} x_{j} \notin E\left(G^{*}\right)} d_{G^{*}}\left(x_{i}\right) d_{G^{*}}\left(x_{j}\right) \\
& =\sum_{v_{i} v_{j} \notin E(G)}\left[2 d_{G}\left(v_{i}\right)\right] \cdot\left[2 d_{G}\left(v_{j}\right)\right] \\
& =4 \sum_{v_{i} v_{j} \notin E(G)} d_{G}\left(v_{i}\right) d_{G}\left(v_{j}\right) \\
& =4 \bar{M}_{2}(G) .
\end{aligned}
$$

The total contribution of nonadjacent vertex pairs of type 2 to $\bar{M}_{1}\left(G^{*}\right)$ and $\bar{M}_{2}\left(G^{*}\right)$ are, respectively, given by

$$
\begin{aligned}
\sum_{i=1}^{n}\left[d_{G^{*}}\left(x_{i}\right)+d_{G^{*}}\left(y_{i}\right)\right] & =\sum_{i=1}^{n}\left[2 d_{G}\left(v_{i}\right)+2 d_{G}\left(v_{i}\right)\right] \\
& =4 \sum_{i=1}^{n} d_{G}\left(v_{i}\right) \\
& =8 m
\end{aligned}
$$

and

$$
\begin{aligned}
\sum_{i=1}^{n} d_{G^{*}}\left(x_{i}\right) d_{G^{*}}\left(y_{i}\right) & =\sum_{i=1}^{n}\left[2 d_{G}\left(v_{i}\right)\right]\left[2 d_{G}\left(v_{i}\right)\right] \\
& =4 \sum_{i=1}^{n}\left[d_{G}\left(v_{i}\right)\right]^{2} \\
& =4\left[\sum_{i=1}^{n} d_{G}\left(v_{i}\right)\right]^{2}-8 \sum_{i=1}^{n} \sum_{j=1, j \neq i}^{n} d_{G}\left(v_{i}\right) d_{G}\left(v_{j}\right) \\
& =16 m^{2}-8 M_{2}(G)-8 \bar{M}_{2}(G) .
\end{aligned}
$$

Now, we consider the total contribution of nonadjacent vertex pairs of type 3 to $\bar{M}_{1}\left(G^{*}\right)$ and $\bar{M}_{2}\left(G^{*}\right)$, respectively.

For each $x_{i}$, there exist $n-1-d_{G}\left(v_{i}\right)$ vertices in the set $\left\{y_{1}, \ldots, y_{n}\right\}$, among which every vertex together with $x_{i}$ compose a nonadjacent vertex pairs of $G^{*}$. The total contribution of these $n-1-d_{G}\left(v_{i}\right)$ nonadjacent vertex pairs to $\bar{M}_{1}\left(G^{*}\right)$ is $\sum_{x_{i} y_{j} \notin E\left(G^{*}\right)}\left[d_{G^{*}}\left(x_{i}\right)+\right.$ $\left.d_{G^{*}}\left(y_{j}\right)\right]=\sum_{v_{i} v_{j} \notin E(G)}\left[2 d_{G}\left(v_{i}\right)+2 d_{G}\left(v_{j}\right)\right]=2 \bar{D}_{G}^{1}\left(v_{i}\right)$ and to $\bar{M}_{2}\left(G^{*}\right)$ is $\sum_{x_{i} y_{j} \notin E\left(G^{*}\right)}\left[d_{G^{*}}\left(x_{i}\right) d_{G^{*}}\left(y_{j}\right)\right]=4 \sum_{v_{i} v_{j} \notin E(G)} d_{G}\left(v_{i}\right) d_{G}\left(v_{j}\right)=4 \bar{D}_{G}^{2}\left(v_{i}\right)$.

So we have

$$
\begin{aligned}
\sum_{i \neq j ; x_{i} y_{j} \notin E\left(G^{*}\right)}\left[d_{G^{*}}\left(x_{i}\right)+d_{G^{*}}\left(y_{j}\right)\right] & =\sum_{i=1}^{n} 2 \bar{D}_{G}^{1}\left(v_{i}\right) \\
& =2 \sum_{i=1}^{n} \bar{D}_{G}^{1}\left(v_{i}\right) \\
& =4 \bar{M}_{1}(G) \quad \text { (by Eq. (1)) }
\end{aligned}
$$


and

$$
\begin{aligned}
\sum_{i \neq j ; x_{i} y_{j} \notin E\left(G^{*}\right)}\left[d_{G^{*}}\left(x_{i}\right) d_{G^{*}}\left(y_{j}\right)\right] & =\sum_{i=1}^{n} 4 \bar{D}_{G}^{2}\left(v_{i}\right) \\
& =8 \bar{M}_{2}(G) \quad(\text { by Eq. (2)). }
\end{aligned}
$$

Therefore,

$$
\begin{aligned}
\bar{M}_{1}\left(G^{*}\right)= & \sum_{x_{i} x_{j} \notin E\left(G^{*}\right)}\left[d_{G^{*}}\left(x_{i}\right)+d_{G^{*}}\left(x_{j}\right)\right]+\sum_{y_{i} y_{j} \notin E\left(G^{*}\right)}\left[d_{G^{*}}\left(y_{i}\right)+d_{G^{*}}\left(y_{j}\right)\right]+ \\
& \sum_{i=1}^{n}\left[d_{G^{*}}\left(x_{i}\right)+d_{G^{*}}\left(y_{i}\right)\right]+\sum_{i \neq j ; x_{i} y_{j} \notin E\left(G^{*}\right)}\left[d_{G^{*}}\left(x_{i}\right)+d_{G^{*}}\left(y_{j}\right)\right] \\
= & 8 \bar{M}_{1}(G)+8 m
\end{aligned}
$$

and

$$
\begin{aligned}
\bar{M}_{2}\left(G^{*}\right)= & \sum_{x_{i} x_{j} \notin E\left(G^{*}\right)} d_{G^{*}}\left(x_{i}\right) d_{G^{*}}\left(x_{j}\right)+\sum_{y_{i} y_{j} \notin E\left(G^{*}\right)} d_{G^{*}}\left(y_{i}\right) d_{G^{*}}\left(y_{j}\right)+ \\
& \sum_{i=1}^{n} d_{G^{*}}\left(x_{i}\right) d_{G^{*}}\left(y_{i}\right)+\sum_{i \neq j ; x_{i} y_{j} \notin E\left(G^{*}\right)} d_{G^{*}}\left(x_{i}\right) d_{G^{*}}\left(y_{j}\right) \\
= & 8 \bar{M}_{2}(G)+\left(16 m^{2}-8 M_{2}(G)-8 \bar{M}_{2}(G)\right)+8 \bar{M}_{2}(G) \\
= & 8 \bar{M}_{2}(G)-8 M_{2}(G)+16 m^{2}
\end{aligned}
$$

This completes the proof.

Now, we give two examples as applications of Theorem 1.
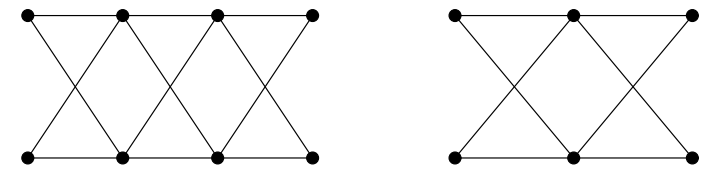

Fig. 2. The graphs $G_{2 n}$.

2.2. Example. Consider Zagreb coindices of the graph $G_{2 n}$, as shown in Fig. 2 .

It can be easily seen that $G_{2 n}$ is just the double graph of the $n$-vertex path $P_{n}$. By an elementary calculation, we obtained $\bar{M}_{1}\left(P_{n}\right)=2(n-2)^{2}, \bar{M}_{2}\left(P_{n}\right)=2 n^{2}-10 n+13$ and $M_{2}\left(P_{n}\right)=4 n-8$. It then follows from Theorem 1 that $\bar{M}_{1}\left(G_{2 n}\right)=8 \times 2(n-2)^{2}+$ $8(n-1)=16 n^{2}-56 n+56$ and $\bar{M}_{2}\left(G_{2 n}\right)=8\left(2 n^{2}-10 n+13\right)-8(4 n-8)+16(n-1)^{2}=$ $32 n^{2}-144 n+184$.

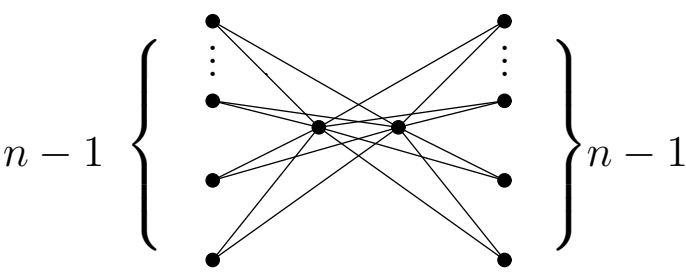

Fig. 3. The graphs $H_{2 n}$.

2.3. Example. Consider Zagreb coindices of the graph $H_{2 n}$, as shown in Fig. 3 . 
It can be easily seen that $H_{2 n}$ is just the double graph of the $n$-vertex star $S_{n}$. By an elementary calculation, we obtained $\bar{M}_{1}\left(S_{n}\right)=n^{2}-3 n+2, \bar{M}_{2}\left(S_{n}\right)=\frac{1}{2}\left(n^{2}-3 n+2\right)$ and $M_{2}\left(S_{n}\right)=(n-1)^{2}$. It then follows from Theorem 1 that $\bar{M}_{1}\left(H_{2 n}\right)=8\left(n^{2}-3 n+\right.$ $2)+8(n-1)=8 n^{2}-16 n+8$ and $\bar{M}_{2}\left(H_{2 n}\right)=8\left[\frac{1}{2}\left(n^{2}-3 n+2\right)\right]-8(n-1)^{2}+16(n-1)^{2}=$ $12 n^{2}-28 n+16$.

Now, we give formulas for Zagreb coindices of $k$-iterated double graphs.

2.4. Theorem. Let $G$ be a nontrivial graph of order $n$ and size $m$, and let $G^{k *}$ be its $k$-th iterated double graph. Then

(i) $\bar{M}_{1}\left(G^{k *}\right)=8^{k} \bar{M}_{1}(G)+2^{2 k+1}\left(2^{k}-1\right) m$;

(ii) $\bar{M}_{2}\left(G^{k *}\right)=8^{k} \bar{M}_{1}(G)-\left[8^{k}\left(2^{k}-1\right)\right] M_{2}(G)+2\left[8^{k}\left(2^{k}-1\right)\right] m^{2}$.

Proof. For any nontrivial graph $G$ with $n$ vertices and $m$ edges, the number of vertices in $G^{*}$ is $2 n$ and the number of edges in $G^{*}$ equals to $2 m$ plus those edges between the sets $\left\{x_{1}, \ldots, x_{n}\right\}$ and $\left\{y_{1}, \ldots, y_{n}\right\}$, that is, $2 m+\sum_{i=1}^{n} d_{G}\left(v_{i}\right)=4 m$.

Now, we can deduce that $G^{k *}$ has $2^{k} n$ vertices and $4^{k} m$ edges.

By Theorem 1 and the definition of $k$-th iterated double graph, for $k \geq 1$, we have

$$
\bar{M}_{1}\left(G^{k *}\right)=8 \bar{M}_{1}\left(G^{(k-1) *}\right)+8 \cdot\left(4^{k-1} m\right)=8 \bar{M}_{1}\left(G^{(k-1) *}\right)+2^{2 k+1} m .
$$

By the recursive relations (4), we have

$$
\begin{aligned}
\bar{M}_{1}\left(G^{k *}\right) & =8 \bar{M}_{1}\left(G^{(k-1) *}\right)+2^{2 k+1} m \\
& =8\left[8 \bar{M}_{1}\left(G^{(k-2) *}\right)+2^{2(k-1)+1} m\right]+2^{2 k+1} m \\
& =8^{2} \bar{M}_{1}\left(G^{(k-2) *}\right)+2^{2 k+2} m+2^{2 k+1} m \\
& =8^{3} \bar{M}_{1}\left(G^{(k-3) *}\right)+2^{2 k+3} m+2^{2 k+2} m+2^{2 k+1} m \\
& =\cdots \\
& =8^{k} \bar{M}_{1}\left(G^{(0) *}\right)+2^{3 k} m+\ldots+2^{2 k+3} m+2^{2 k+2} m+2^{2 k+1} m \\
& =8^{k} \bar{M}_{1}(G)+2^{2 k+1}\left(2^{k}-1\right) m .
\end{aligned}
$$

Let us proceed to $(i i)$. By Theorem 1 and the definition of $k$-th iterated double graph, for $k \geq 1$, we have

$$
\bar{M}_{2}\left(G^{k *}\right)=8 \bar{M}_{2}\left(G^{(k-1) *}\right)-8 M_{2}\left(G^{(k-1) *}\right)+16\left(4^{k-1} m\right)^{2}
$$

that is,

$$
\bar{M}_{2}\left(G^{k *}\right)=8 \bar{M}_{2}\left(G^{(k-1) *}\right)-8 M_{2}\left(G^{(k-1) *}\right)+16^{k} m^{2} .
$$

By a similar argument to that employed in Theorem 1 to treat the second Zagreb coindex and using Eq. (3) at the same time, we obtain $\sum_{x_{i} y_{j} \in E\left(G^{*}\right)} d_{G^{*}}\left(x_{i}\right) d_{G^{*}}\left(y_{j}\right)=$ $4 \sum_{i=1}^{n} D_{G}^{2}\left(v_{i}\right)=8 M_{2}(G)$. 
In view of this equality, we obtain

$$
\begin{aligned}
M_{2}\left(G^{*}\right)= & \sum_{x_{i} x_{j} \in E\left(G^{*}\right)} d_{G^{*}}\left(x_{i}\right) d_{G^{*}}\left(x_{j}\right)+\sum_{y_{i} y_{j} \in E\left(G^{*}\right)} d_{G^{*}}\left(y_{i}\right) d_{G^{*}}\left(y_{j}\right)+ \\
& \sum_{x_{i} y_{j} \in E\left(G^{*}\right)} d_{G^{*}}\left(x_{i}\right) d_{G^{*}}\left(y_{j}\right) \\
= & 4 \sum_{v_{i} v_{j} \in E(G)} d_{G}\left(v_{i}\right) d_{G}\left(v_{j}\right)+4 \sum_{v_{i} v_{j} \in E(G)} d_{G}\left(v_{i}\right) d_{G}\left(v_{j}\right)+ \\
& \sum_{x_{i} y_{j} \in E\left(G^{*}\right)} d_{G^{*}}\left(x_{i}\right) d_{G^{*}}\left(y_{j}\right) \\
= & 8 M_{2}(G)+8 M_{2}(G) \\
= & 16 M_{2}(G) .
\end{aligned}
$$

So we have the recursive relation $M_{2}\left(G^{k *}\right)=16 M_{2}\left(G^{(k-1) *}\right)$ for each $k \geq 1$, and then

$$
M_{2}\left(G^{k *}\right)=16^{k} M_{2}\left(G^{(0) *}\right)=16^{k} M_{2}(G) .
$$

By Eqs. (5) and (6), we obtain

$$
\begin{aligned}
\bar{M}_{2}\left(G^{k *}\right)= & 8 \bar{M}_{2}\left(G^{(k-1) *}\right)-8 \cdot 16^{k-1} M_{2}(G)+16^{k} m^{2} \\
= & 8\left[8 \bar{M}_{2}\left(G^{(k-2) *}\right)-8 \cdot 16^{k-2} M_{2}(G)+16^{k-1} m^{2}\right]-8 \cdot 16^{k-1} M_{2}(G)+16^{k} m^{2} \\
= & 8^{2} \bar{M}_{2}\left(G^{(k-2) *}\right)-8^{2} \cdot 16^{k-2} M_{2}(G)-8 \cdot 16^{k-1} M_{2}(G)+8 \cdot 16^{k-1} m^{2}+16^{k} m^{2} \\
= & \cdots \\
= & \left.8^{k} \bar{M}_{1}\left(G^{(0) *}\right)-\left[8 \cdot 16^{k-1}+8^{2} \cdot 16^{k-2}+\ldots+8^{k} \cdot 16^{0}\right)\right] M_{2}(G)+ \\
& {\left[16^{k}+8 \cdot 16^{k-1}+\ldots+8^{k-1} \cdot 16^{1}\right] m^{2} } \\
= & \left.8^{k} \bar{M}_{1}(G)-\left[8 \cdot 16^{k-1}+8^{2} \cdot 16^{k-2}+\ldots+8^{k} \cdot 16^{0}\right)\right] M_{2}(G)+ \\
& {\left[16^{k}+8 \cdot 16^{k-1}+\ldots+8^{k-1} \cdot 16^{1}\right] m^{2} } \\
= & 8^{k} \bar{M}_{1}(G)-\left[8^{k}\left(2^{k}-1\right)\right] M_{2}(G)+2\left[8^{k}\left(2^{k}-1\right)\right] m^{2},
\end{aligned}
$$

as claimed.

\section{References}

[1] A.R. Ashrafi, T. Došlić, A. Hamzeh, The Zagreb coindices of graph operations, Discrete Appl. Math., 158 (2010) 1571-1578.

[2] A.R. Ashrafi, T. Došlić, A. Hamzeh, Extremal graphs with respect to the Zagreb coindices, MATCH Commun. Math. Comput. Chem., 65 (2011) 85-92.

[3] A. Behtoei, M. Jannesari, B. Taeri, Maximum Zagreb index, minimum hyper-Wiener index and graph connectivity, Appl. Math. Lett., 22 (2009) 1571-1576.

[4] H. Hua, Zagreb $M_{1}$ index, indenpedence number and connectivity in graphs, $M A T C H$ Commun. Math. Comput. Chem., 60 (2008) 45-56.

[5] H. Hua, S. Zhang, Relations between Zagreb coindices and some distance-based topological indices, MATCH Commun. Math. Comput. Chem., 68 (2012) 199-208.

[6] H. Hua, On the degree distance of some composite graphs, Bull. Aust. Math. Soc., 85 (2012) 164-171. 
[7] H. Hua, S. Zhang, K. Xu, Further results on the eccentric distance sum, Discrete Appl. Math., 160 (2012) 170-180.

[8] M.H. Khalifeh, H. Yousefi-Azari, A.R. Ashrafi, The first and second Zagreb indices of graph operations, Discrete Appl. Math., 157 (2009) 804-811.

[9] E. Munarini, C. Perelli Cippo, A. Scagliola, N. Zagaglia Salvi, Double graphs, Discrete Math., 308 (2008) 242-254.

[10] P.S. Ranjini, V. Lokesha, I.N. Cangül, On the Zagreb indices of the line graphs of the subdivision graphs, Appl. Math. Comput., 218 (2011) 699-702.

[11] R. Todeschini, V. Consoni, Handbook of Molecular Descriptors, Wiley-VCH, New York, 2002.

[12] J. Wang, Q. Ma, Some results on edge cover coloring of double graphs, Appl. Math., 3 (2012) 264-266.

[13] L. Xu, Y. Hou, Equienergetic bipartite graphs, MATCH Commun. Math. Comput. Chem., 57 (2007) 363-370.

[14] K. Xu, The Zagreb indices of graphs with a given clique number, Appl. Math. Lett., 24 (2011) 1026-1030. 